EPJ Web of Conferences 41, 05013 (2013)

DOI: $10.1051 /$ epjconf/20134105013

(C) Owned by the authors, published by EDP Sciences, 2013

\title{
Ultrafast Measurements of Coherent Vibrations in Benzenethiol Monolayer Film
}

\author{
K. Shudo ${ }^{1 \dagger, a}$, K. Doi ${ }^{1 \ddagger}$, I. Katayama ${ }^{1 \dagger}$, M. Kitajima ${ }^{2}$, J. Takeda ${ }^{1 \dagger}$ \\ 1 Faculty of Science/Engineering ${ }^{\dagger}$ and Graduate School of Engineering ${ }^{\ddagger}$, \\ Yokohama National University, Hodogaya-ku Tokiwadai 79-5, Yokohama 240-8501 Japan \\ 2 School of Applied Sciences, National Defense Academy, \\ Hashirimizu 1-10-20, Yokosuka 239-8686, Japan
}

\begin{abstract}
Ultrafast photo-reflectance at a monolayer film of benzentiol molecule adsorbed on a gold surface was measured by means of pump-probe method using an ultrashort pulse laser. The surface-sensitive transient response in THz region revealed coherent motion of the molecules affected by the adsorption.
\end{abstract}

\section{Introduction}

Among organic molecules, thiol family is known to easily form a self-assembled monolayer (SAM) film with a bond at the sulfer site to even noble metal surfaces. A benzentiol (BT) molecule contains the most fundamental aromatic functional that offers a variety of applications [1]. As molecular vibrations of such SAMs are fingerprints of adsorbed structures, we consider the dynamic motion of the molecules to elucidate molecule-substrate interaction in the ultrathin film. Ultrafast measurements of coherent molecular motion at the femtosecond time-scale are available with optical methods using ultrashort-pulsed laser systems. In this research, we measured the transient reflectance signal from $\mathrm{Au}$ surface, on which the SAM of BT was adsorbed at the monolayer thickness. The resultant spectra in frequency domain gave information on coherent motions of the adsorbed layer.
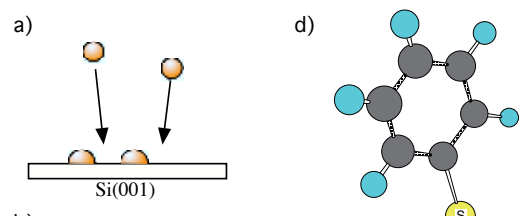

b)

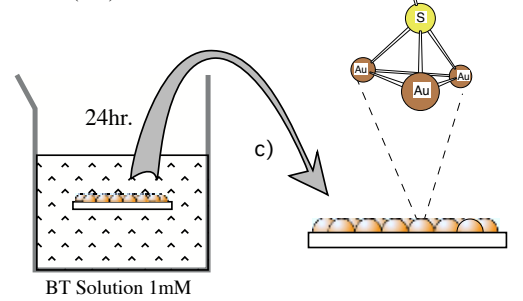

Fig. 1. Schematics of preparation of SAM sample. (a) Au films are formed on $\mathrm{Si}$, (b) then soaked into BT solution, and (c) finally dried slowly in air after rinse with purified water. On the surface of the gold, BT was adsorbed as in (d).

\footnotetext{
${ }^{a}$ Corresponding authos's e-mail: ken1@ynu.ac.jp
}

This is an Open Access article distributed under the terms of the Creative Commons Attribution License 2.0, which permits unrestricted use, distribution, and reproduction in any medium, provided the original work is properly cited. 


\section{Experimental}

Strips of silicon (001) wafer were used as a substrate material. Prior to optical measurements the specimen were treated as follows (See Fig.1). Gold ( $\mathrm{Au}$ ) atoms were attached to the surface by sputter deposition to form Au films at thickness of a few Angstrom to several nm. The specimen was soaked into ethanol solusion of BT (1 mM) for $24 \mathrm{hrs}$. On the Au surface, BT molecules are adsorbed at a monolayer thickness [2]. The surface morphology of Au was confirmed with a atomic-force microscopes.

At the surface of the specimen, optical response was measured by means of pump-probe electrooptical (EO) sampling technique. Ultrashort pulse (7.5 fs) of Ti:sapphire laser ( $800 \mathrm{~nm})$ was used as a light source. A pump beam was irradiated from the direction of the surface normal, and it was focused at the surface. A probe pulse delayed variably from the pump pulse was focused to the surface area where the pump was irradiated. The reflected probe beam was divided into two polarized components orthogonal to each other. The difference of the intensities of the two components, measured with a pair of photodiodes, was detected electrically, and it was recorded as the signal of asymmetry in the optical reflectance due to the surface deformation.

\subsection{Result and Discussion}

The transient signal of BT monolayer film was plotted along the varied delay time as the inset in Fig. 2. After intense signal at the beginning (where 0 delay at $0.37 \mathrm{ps}$ ), a slow decay was observed. After subtraction of the decay, a complex oscillatory signal was left (shown in the main panel). In the Fourier transformed spectra (Fig. 3) from the residual signal, several peaks are found. In the figure, we also plotted peaks of vibrational components of liquid BT (> 98\% purity) obtained with conventional CW-Raman spectroscopy.

Approximately, the most of the peaks in the Fourier spectra (gray in Fig. 3) correspond to the vibrational peaks in the conventional spectra (black in Fig. 3). No peak of Si substrate was observed in the Fourier spectra because we can choose polarization of the incident beam to excite the surface layer selectively, based on a selection rule associated with photoexcitation [3]. On the other hand, a peak originated from crystalline $\mathrm{Si}(\sim 16 \mathrm{THz})$ appeared as the strongest signal in CW-Raman (data not

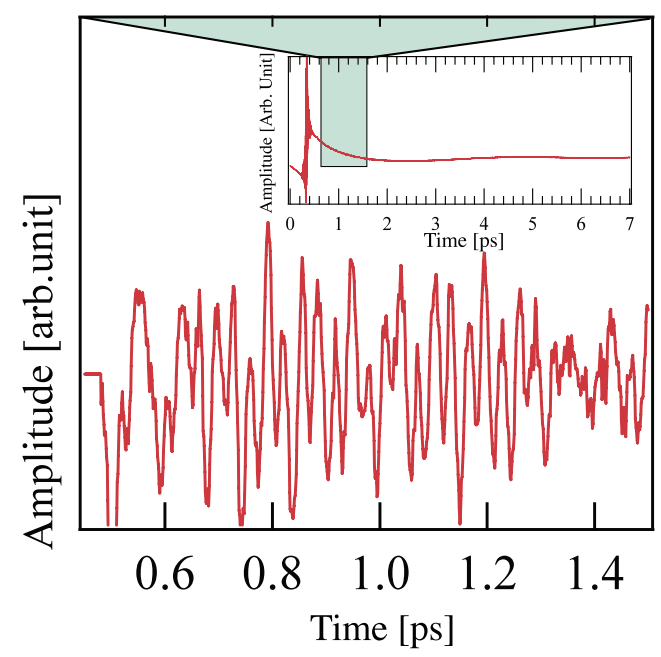

Fig. 2. Magnification of real-time transient response, plotted after subtraction of a slow component. The inset is an observed transient response of the surface asymmetry in reflectance (EO sampling; see Text). The shadowed area in the inset corresponds to the main panel. 


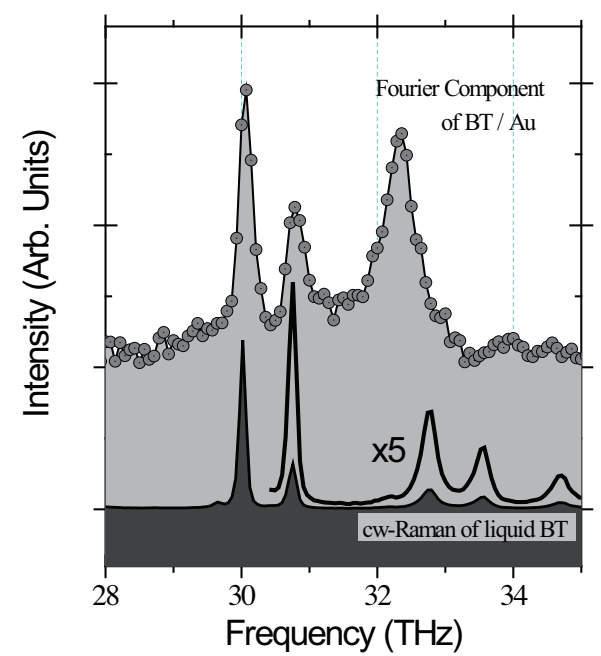

Fig. 3. Fourier components of the subtracted signal (gray) in Fig. 2. A range with typical peaks is only shown. In comparison, a conventional Raman spectra of liquid BT is shown (black), with a maginified curve of five times..

shown). Thus, we can clarify the coherent motion of the two-dimensional structure of the BT monolayer selectively, since the ultrashort pulses excite the SAM film impulsively. There are some discrepancies in the peak positions between the SAM film and the liquid phase. These shifts in the vibration can be explained in terms of immobilization of the molecule at Au surface [1], as in Fig. 1(d). We calculated electronic and vibrational eigenvalues with first-principle method, and the vibronic modes of the BT adsorbed on the surface are in good agreement with the experimental results. The relative intensity of each mode varies when the thickness of Au deposition, indicating surface-enhanced Raman scattering (SERS) effect is active at the surface layer [4]. Morphology of Au surface and alignment of molecules seem to affect the ultrafast dynamics; the ultrafast pump-and-probe measurement enables us to discuss dynamic phenomena of real-time coherent motion in the monolayer thin films.

\subsection{Conclusion}

Using pump-probe EO sampling technique, it is possible to selectively and sensitively measure the transient optical asymmetry originated from the vibration of organic molecules adsorbed on surfaces. To discuss the intermolecular, nonliner, and low-dimentional lattice interaction in such molecular systems, real-time analysis will give new information in terms of time-dependent intensity, phase and beat of each vibronic mode.

\section{References}

1. R.C. Price, and R.L. Whetten, "All-aromatic, nanometer-scale, gold-cluster thiolate complexes", J. Am. Chem. Soc. 2005,13750-13751 (2005).

2. H. Kang, T. Park, I. Choi, Y. Lee, E. Ito, M. Hara, J. Noh, "Formation of large ordered domains in benzenethiol self-assembled monolayers on $\mathrm{Au}(111)$ observed by scanning tunneling microscopy", Ultramicroscopy 109, 1011-1014 (2009).

3. M. Hase, M. Kitajima, A-M. Constantinescu, and H. Petek, "The birth of a quasiparticle in silicon observed in time-frequency space", Nature426, 51-54 (2003).

4. I. Katayama, S. Koga, K. Shudo,J. Takeda, T. Shimada, A. Kubo, S. Hishita, D. Fujita, and M. Kitajima, "Ultrafast Dynamics of Surface-enhanced Raman Scattering due to Au Nanostructures", Nano Letters 11, 2648-2654 (2011). 